\title{
ONE PARAMETER FAMILIES OF RIEMANN SURFACES OF GENUS TWO
}

\author{
ANTONIO F. COSTA \\ Departamento de Matemáticas Fundamentales, Facultad de Ciencias, U.N.E.D. 28040-Madrid, Spain \\ e-mail: acosta@mat.uned.es \\ and GONZALO RIERA \\ P.Universidad Católica, Casilla 306, Correo 22, Santiago, Chile
}

(Received 6 October, 1999)

\begin{abstract}
We obtain the complex orbifold structure of the moduli space for one parameter equisymmetric Riemann surfaces of genus two. For each family, by using the orbifold structure, we obtain the points in the moduli corresponding to real algebraic curves and a special form for the period matrices of Riemann surfaces that admit an anticonformal involution. We describe the topological type of anticonformal involutions admitted by surfaces of the families depending on the type of period matrix.
\end{abstract}

1991 Mathematics Subject Classification. 14H45, 30F45.

1. Introduction. One of the most important problems in the theory of Riemann surfaces is to determine when two surfaces are conformally equivalent. This problem is completely solved for symmetric Riemann surfaces (i.e. real algebraic curves) of genus two in [16]. To deal with this question one considers a basis of the homology and a corresponding basis of holomorphic differentials; the period matrices lie in Siegel's space, and two matrices are equivalent under the modular group if and only if the surfaces are conformally equivalent. This procedure not only deals with the equivalence problem but also it endows the moduli space with a geometrical structure. We shall focus our attention on such a structure. The moduli space has the structure of a complex orbifold (i.e. an orbifold with complex analytic transition mappings), since the modular group acts properly discontinuously on the complex space of period matrices. In this work we shall describe the orbifold structure for two uniparametric families of genus two surfaces and we shall use such a description to yield the points in the moduli space corresponding to symmetric surfaces.

For example, for $g=1$ the set of period matrices for elliptic curves is $\{\tau: \operatorname{Im}(\tau)>0\}$. Two elliptic surfaces with period matrices $\tau$ and $\tau^{*}$ are conformally equivalent if and only if there exists an element $\left(\begin{array}{ll}a & b \\ c & d\end{array}\right)$ in $\operatorname{PSL}(2, \mathbf{Z})$ such that $\tau^{*}=\frac{a \tau+b}{c \tau+d}$. The moduli space with the structure of a complex orbifold is of complex dimension one and genus 0 . It has two cone points with isotropy cyclic groups of orders 2 and 3 and a cusp. The cone points are given by the classes $[i]$ and $\left[\frac{1}{2}+\frac{\sqrt{3}}{2} i\right]$ and correspond to Platonic surfaces; i.e. Riemann surfaces admitting a representation by generalized regular polyhedra or regular maps (see [8]). We shall denote by $[\infty]$ the cusp. On this orbifold there is an antianalytic involution given by the conjugation $c$, whose fixed point set is an arc which passes through the two singular points and has its ends at $[\infty]$. The points in $\operatorname{Fix}(c)$ correspond to Riemann surfaces 
given by real elliptic curves. The $\operatorname{arc}$ in $\operatorname{Fix}(c)$ joining $[i]$ to $[\infty]$ corresponds to real elliptic curves which are uniformised by real rectangular lattices and whose real part has two connected components. The arc from $[i]$ to $\left[\frac{1}{2}+\frac{\sqrt{3}}{2} i\right]$ and the one from $\left[\frac{1}{2}+\frac{\sqrt{3}}{2} i\right]$ to $[\infty]$ consist of Riemann surfaces uniformised by real rhombic lattices and whose real parts have one connected component; (see [1], [5] and [9]).

The moduli space of surfaces of genus two has complex dimension 3 and a description analogous to the genus one case is not possible. A way to produce nice descriptions is to restrict oneself to equisymmetric surfaces. For example the equisymmetric strata of dimension zero have been studied by R. Kulkarni [10]. We consider here the complex dimension one equisymmetric strata in the moduli space of genus two surfaces.

The symmetric genus two Riemann surfaces and their period matrices are well known; see for example [2], [14], [11]. In the moduli space of surfaces of genus two there are two 1-dimensional complex suborbifolds corresponding to two 1-parameter families of equisymmetric Riemann surfaces; (see [2]). The automorphism groups of the surfaces in the first family contain the group $D_{4}$ and this family consists of the algebraic curves $y^{2}=x\left(x^{2}-1\right)(x-\mu)\left(x-\mu^{-1}\right)$, where $\mu \neq \pm 1,0$. The automorphism groups in the second family contain $D_{6}$ and the curves are defined by the equations $y^{2}=x(x-1)(x-\lambda)\left(x-\frac{\lambda-1}{\lambda}\right)\left(x-\frac{1}{1-\lambda}\right)$, where $\lambda \neq \frac{1 \pm \sqrt{3} i}{2}, 0,1$.

For each family we describe the corresponding family of Jacobians and modular groups and we obtain the orbifold structure of the moduli space in each case. Let $[p, q, \infty]$ be the hyperbolic triangle in the complex upper half plane with one vertex at infinity and angles at the finite vertices of $\pi / p$ and $\pi / q$. We shall call $O_{p, q, \infty}$ the 1-complex orbifold structure of the orbifold obtained by identifying two triangles $[p, q, \infty]$ by the identity in the boundary. Note that the complex orbifold structure is uniquely determined by the numbers $p$ and $q$. Adding to the first family the elliptic curve $y^{2}=x(x+1)(x-1)^{3}$, and to the second family the elliptic curve $y^{2}=x(x-1)\left(x-\frac{1+\sqrt{3} i}{2}\right)^{3}$, we obtain that the complex orbifolds for the moduli space of each are of type $O_{p, q, \infty}$. The orbifold is $O_{2,4, \infty}$ for the first family and $O_{2,6, \infty}$ for the second one. The two cone points in the first family correspond to the Platonic surface of genus 2 with automorphism groups of order 48 and the elliptic curve $y^{2}=x(x+1)(x-1)^{3}$. The cone points in the second family correspond to the surface with 24 automorphisms and the elliptic curve $y^{2}=x(x-1)\left(x-\frac{1+\sqrt{3} i}{2}\right)^{3}$.

For the two families, the anticonformal involution $c$, given by conjugation, has as its fixed point set $\operatorname{Fix}(c)$, the points corresponding to curves possessing a real form; i.e. Riemann surfaces which possess an anticonformal involution. In the two cases $\operatorname{Fix}(c)$ is an arc with its ends at the cusp and which passes through the cone points. We describe the location of the real algebraic curves in the moduli space of the complex algebraic curves for each family. We obtain the types of symmetries admitted for each surface depending on the corresponding point in $\operatorname{Fix}(c)$.

2. Equisymmetric families of surfaces of genus two. A compact Riemann surface of genus two is given by an hyperelliptic equation with six branch points $\{0,1, \infty$, $a, b, c\}: y^{2}=x(x-1)(x-a)(x-b)(x-c)$. Since every automorphism commutes with the hyperelliptic involution, then such automorphism is given by a Möbius transformation of the Riemann sphere permuting the six branch points. The classification of all possible symmetries is then easy to obtain; the complete list can be found in several papers, for example [2], [14] and [11]. 
Complex parameters

3

2

1

1

0

0

0
Algebraic equation

$$
\begin{gathered}
y^{2}=x(x-1)(x-\lambda)(x-\mu)(x-v) \\
y^{2}=x(x-1)(x-\lambda)(x-\mu)\left(x-\mu \frac{1-\lambda}{1-\mu}\right) \\
y^{2}=x\left(x^{2}-1\right)(x-\mu)\left(x-\mu^{-1}\right) \\
y^{2}=x(x-1)(x-\lambda)\left(x-\frac{\lambda-1}{\lambda}\right)\left(x-\frac{1}{1-\lambda}\right) \\
y^{2}=x\left(x^{2}-1\right)(x-2)\left(x-\frac{1}{2}\right) \\
y^{2}=x\left(x^{4}-1\right) \\
y^{2}=x^{5}-1
\end{gathered}
$$

Order of the automorphisms group

2
4
8
12
24
48
10

Then there are two families of curves depending on one complex parameter; these are the families that we shall study.

3. The moduli space and the modular group for the family $y^{2}=x\left(x^{2}-1\right)$ $(x-\mu)\left(x-\mu^{-1}\right), \mu \neq \pm 1,0$.

We shall call this family $F_{1}$. The Möbius transformation $\bar{\sigma}: x \rightarrow \frac{1}{x}$ lifts to an automorphism $\widehat{\sigma}$ of order 4 on each surface of the family $F_{1}$ and $\bar{\tau}_{\mu}: x \rightarrow \frac{\mu x-1}{x-\mu}$ lifts similarly to an automorphism $\widehat{\tau}$ of order 2 . The group $\langle\widehat{\sigma}, \widehat{\tau}\rangle$ is $D_{4}$.

The family $F_{1}$ passes through two exceptional curves:

- $\mu= \pm 2, \pm \frac{1}{2}$ the curve admitting 24 automorphisms,

- $\mu= \pm i$ the curve admitting 48 automorphisms.

We consider the parametrization $\theta: \mathbf{C}-\{-1,0,1\} \rightarrow F_{1}$ defined by

$$
\mu \rightarrow \theta(\mu)=\left\{(x, y): y^{2}=x\left(x^{2}-1\right)(x-\mu)\left(x-\mu^{-1}\right)\right\} .
$$

The parametrization $\theta$ is not injective. In view of the unicity of the hyperelliptic involution, two parameters $\mu$ and $\mu^{\prime}$ satisfy $\theta(\mu)=\theta\left(\mu^{\prime}\right)$ (the equality sign means isomorphic Riemann surfaces) if and only if there is a Möbius transformation $T$ such that $T\left\{\infty,-1,0,1, \mu, \mu^{-1}\right\}=\left\{\infty,-1,0,1, \mu^{\prime}, \mu^{\prime-1}\right\}$. Given $\mu \neq \pm i$ and $\mu^{\prime}$, we consider the following subgroups of $\operatorname{Aut}(\widehat{\mathbf{C}}): G_{\mu}=\left\langle\bar{\sigma}, \bar{\tau}_{\mu}\right\rangle, G_{\mu^{\prime}}=\left\langle\bar{\sigma}, \bar{\tau}_{\mu^{\prime}}\right\rangle$ and $G=\left\langle z \rightarrow-z, z \rightarrow \frac{1}{z}\right\rangle$.

Lemma 1. Let $T$ be a Möbius transformation satisfying $T\left\{\infty,-1,0,1, \mu, \mu^{-1}\right\}=$ $\left\{\infty,-1,0,1, \mu^{\prime}, \mu^{\prime-1}\right\}$. Then there are $g \in G, g_{\mu} \in G_{\mu}$ and $g_{\mu^{\prime}} \in G_{\mu^{\prime}}$ such that $g_{\mu^{\prime}} \circ T \circ g_{\mu}=g$.

Proof. Given $T$ we can choose $g_{\mu} \in G_{\mu}$ and $g_{\mu^{\prime}} \in G_{\mu^{\prime}}$ such that $T_{1}(\infty)=g_{\mu^{\prime}} \circ T \circ g_{\mu}(\infty)=\infty$. Now $T_{1}$ is a conformal automorphism of $\mathbf{C}$ such that $T\left\{-1,0,1, \mu, \mu^{-1}\right\}=\left\{-1,0,1, \mu^{\prime}, \mu^{\prime-1}\right\}$. By geometrical arguments and the hypothesis $\mu \neq \pm i$ we obtain $T_{1} \in G$.

As a consequence $\theta: \mathbf{C}-\{-1,0,1\} \rightarrow F_{1}$ is the natural quotient map for the action of the group $G$ on $\mathbf{C}-\{-1,0,1\}$. Then the map $\theta$ provides a 1 -complex orbifold structure on the moduli space for this family.

Proposition 2. The moduli space of Riemann surfaces in the family $F_{1}$ is the Riemann sphere without $\{\infty,-1,0,1\}$ quotiented by the action of $z \rightarrow-z$ and $z \rightarrow z^{-1}$, i.e. isomorphic to the orbifold $O_{2, \infty, \infty}$. The cone point of $O_{2, \infty, \infty}$ corresponds to $\mu=i$, i.e. the surface having automorphism group of order 48 , namely $X_{48}$. 
Now we shall construct the moduli space using period matrices. Let $X$ be a surface of the family $F_{1}$ and let $h$ be the hyperelliptic involution. The automorphism $\widehat{\sigma}$ of $X$ induces an involution in the orbifold $X / h$ fixing two singular points (note that $x \rightarrow \frac{1}{x}$ fixes 1 and -1 that are branched points). The automorphism $\widehat{\tau}$ induces in $X / h$ an involution pairwise permuting the singular points. Finally the product of the two involutions has order two so that the quotient $X / D_{4}=X /\langle\widehat{\sigma}, \widehat{\tau}\rangle$ is an orbifold of genus 0 with four singular points with isotropy groups of orders 2, 2, 2 and 4; (see Figure 1).

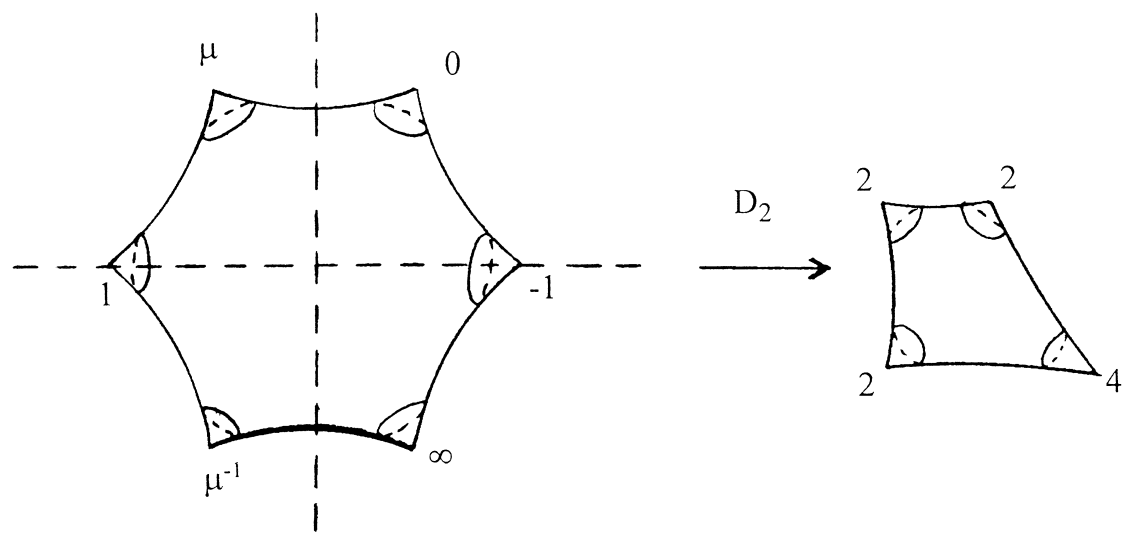

Figure 1

The orbifold $X /\langle\widehat{\sigma}, \widehat{\tau}\rangle$ can be uniformised by a Fuchsian group $\Delta$ of signature $(0 ;[2,2,2,4])$ with fundamental region an hyperbolic quadrilateral $Q$ with angles $\frac{\pi}{2}$, $\alpha, \beta, \gamma ; \alpha+\beta+\gamma=\pi$.
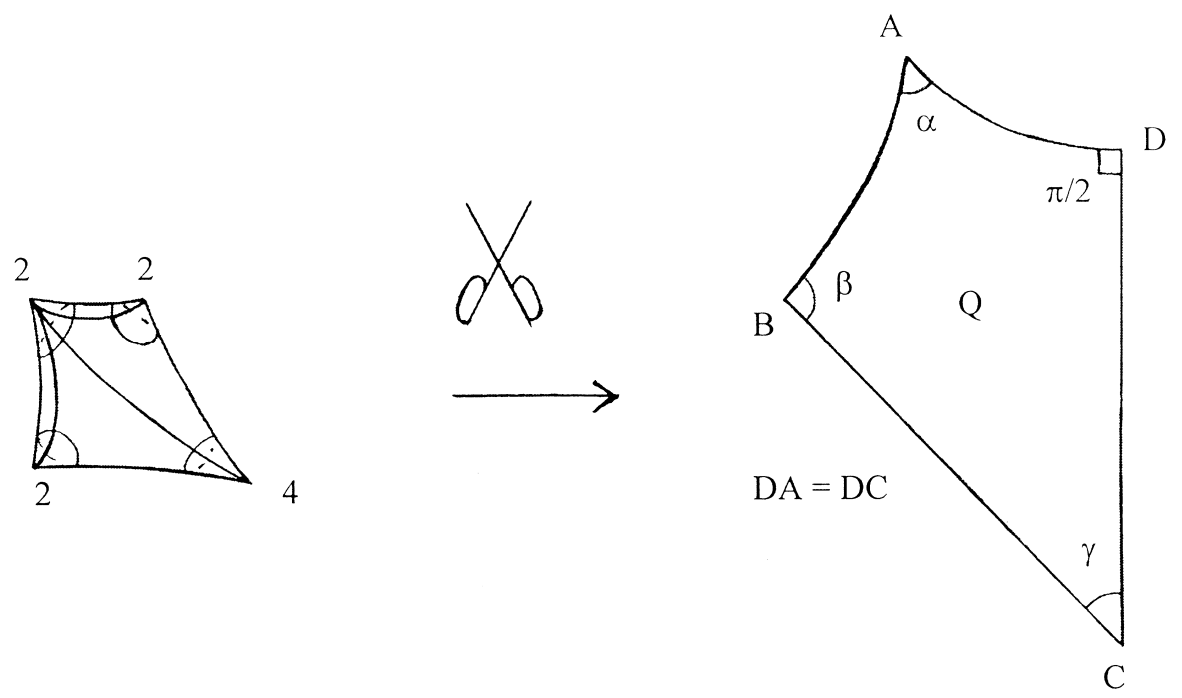

Figure 2

The group $\Delta$ has an elliptic element $\widetilde{\sigma}$ of order four fixing the point $D$ in Figure 2 and projecting on $\widehat{\sigma}$ of $X$. In $\Delta$ there is an elliptic element of order two fixing the middle point of the quadrilateral side $\mathrm{AB}$ and another $\tilde{\tau}$ fixing the middle point of BC. $\tilde{\tau}$ projects on $\widehat{\tau}$ of $X$. 
The fundamental region for a Fuchsian surface group $\Gamma$ uniformising $X$ is obtained by considering 8 images of $Q$ by some representatives of $\Delta / \Gamma \simeq D_{4}$. We obtain a 10-gon divided into 8 quadrilaterals as shown in Figure 3.

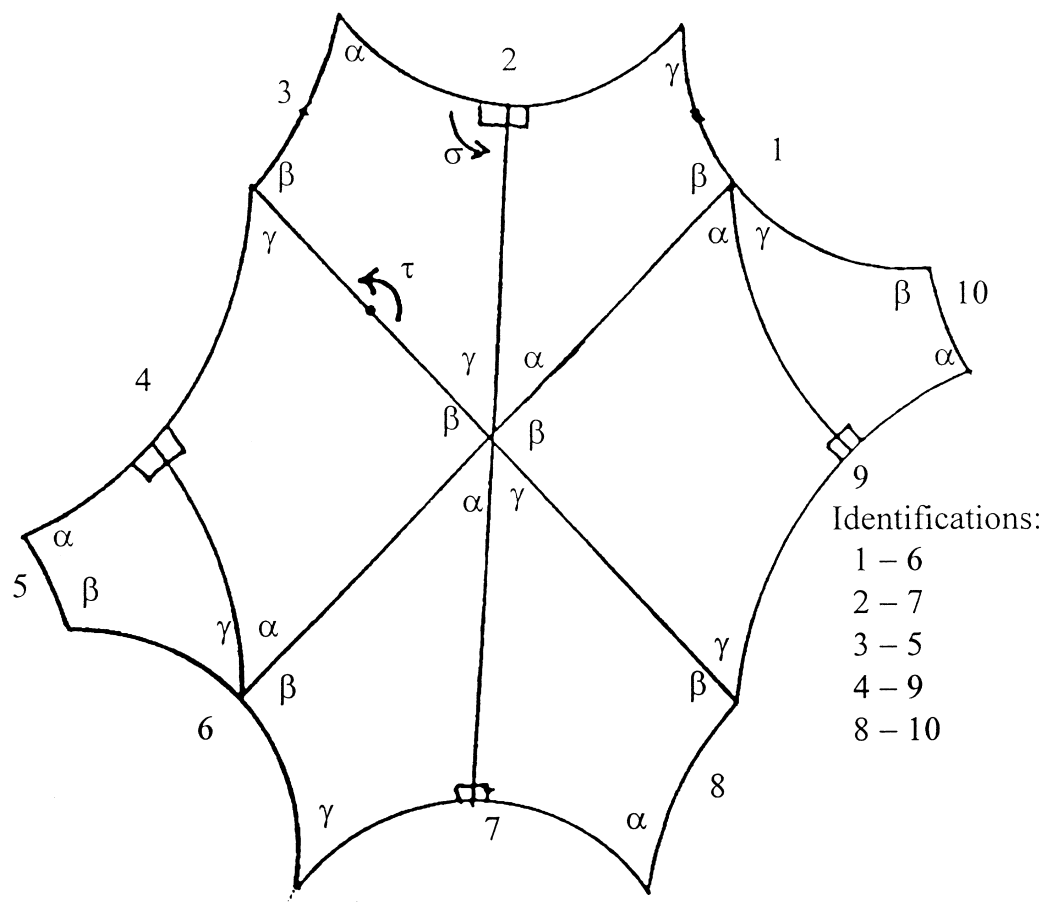

Figure 3

The group $\Gamma$ is generated by hyperbolic elements identifying the side 1 with 6,2 with 7, 3 with 5,4 with 9 and 8 with 10 . The pairwise identification of the polygon sides gives the surface $X$.

The period matrices for the Riemann surfaces of the family have been obtained in [2] but we give here a quicker method. We fix a canonical homology basis as shown in Figure 4.

The basis $\left(e_{1}, e_{2}, e_{3}, e_{4}\right)$ is symplectic. In the terms of this basis the action of the group $D_{4}$ is represented by the matrices

$$
[\sigma]=\left(\begin{array}{cccc}
0 & 1 & -1 & 0 \\
-1 & 0 & 0 & 1 \\
0 & 0 & 0 & 1 \\
0 & 0 & -1 & 0
\end{array}\right),[\tau]=\left(\begin{array}{cccc}
1 & 0 & 0 & -1 \\
0 & -1 & 1 & 0 \\
0 & 0 & 1 & 0 \\
0 & 0 & 0 & -1
\end{array}\right)
$$

If $\left(\begin{array}{ll}A & B \\ C & D\end{array}\right)$ is a symplectic matrix representing the action on the homology of an automorphism, then such an automorphism induces an action on the Siegel space $S_{2}$ of period matrices of surfaces of genus two via $Z^{*}=(A Z+B)(C Z+D)^{-1}$. The set of fixed matrices in $S_{2}$ by the action of $[\sigma]$ and $[\tau]$ is the Jacobian of the family $F_{1}$; (i.e. the Rauch method of computing period matrices of surfaces with automorphisms; see [7]). Then we obtain the matrices $\left\{Z=\frac{1}{2}\left(\begin{array}{cc}t & 1 \\ 1 & t\end{array}\right) ; \operatorname{Im} t>0\right\}$. (See [2], 


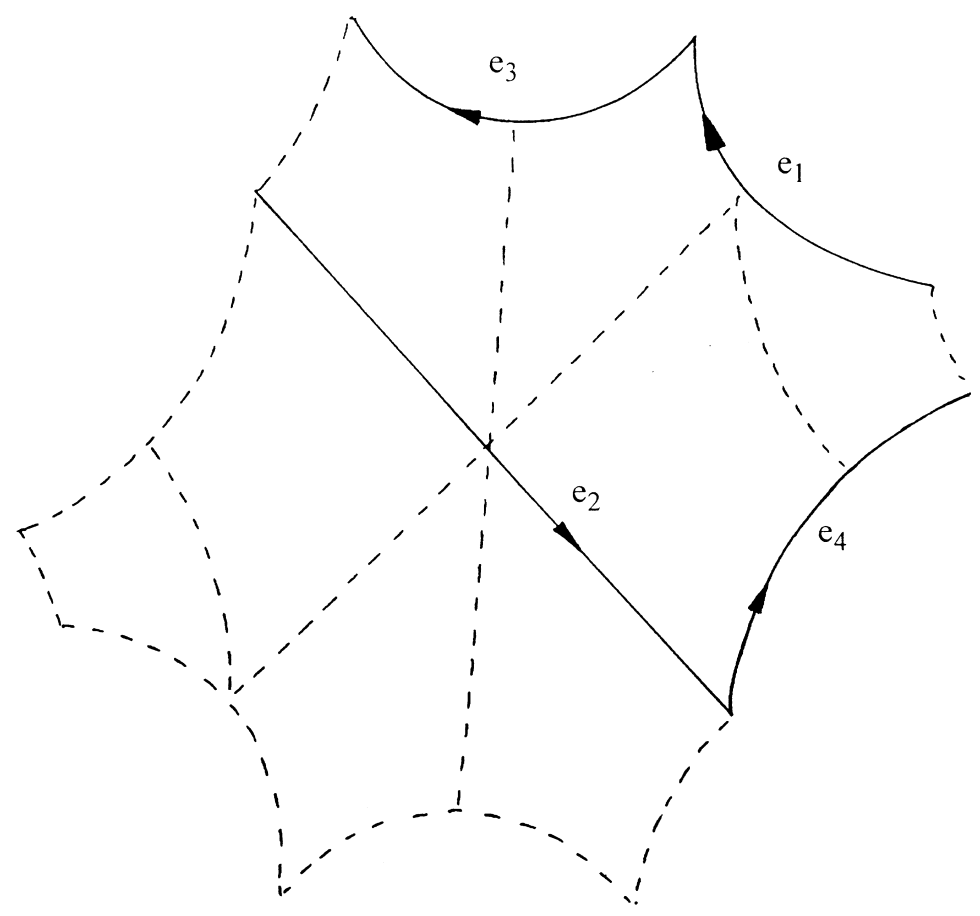

Figure 4

Case III.) We observe that if $Z$ is fixed by $\sigma$, then it is automatically fixed by $\tau$ too. Now we use Theorem 2.1 of [14]. Then the period matrices for the family $F_{1}$ are given by

$$
\begin{gathered}
\Sigma_{1}=\left\{Z=\frac{1}{2}\left(\begin{array}{ll}
t & 1 \\
1 & s
\end{array}\right): \operatorname{Im} t>0\right\}- \\
-\left\{Z=\frac{1}{2}\left(\begin{array}{ll}
t & 1 \\
1 & s
\end{array}\right): \operatorname{Im} t>0, \operatorname{Im} s>0, s=R S(t), R \in \Gamma(2)\right\},
\end{gathered}
$$

where $S$ is the transformation $z \rightarrow-\frac{1}{z}$, and $\Gamma(2)$ is the even modular group. Hence

$$
\Sigma_{1}=\left\{Z=\frac{1}{2}\left(\begin{array}{ll}
t & 1 \\
1 & s
\end{array}\right) ; \operatorname{Im} t>0, t \neq R(i), R \in \Gamma(2)\right\} .
$$

Thus we have obtained the following result.

Proposition 3. The space of period matrices for the family $F_{1}$ is $\Sigma_{1}=\left\{Z=\frac{1}{2}\left(\begin{array}{ll}t & 1 \\ 1 & t\end{array}\right) ; \operatorname{Im} t>0, t \neq R(i), R \in \Gamma(2)\right\}$, where $\Gamma(2)$ is the even modular group.

The matrix $Z=\frac{1}{2}\left(\begin{array}{ll}i & 1 \\ 1 & i\end{array}\right)$ corresponds to the elliptic surface $y^{2}=x(x+1)(x-1)^{3}$, which is the elliptic surface uniformized by the lattice generated by 1 and $i$. 
Let us now obtain the modular group for $\Sigma_{1}$. The modular group is obtained from the matrices $\left(\begin{array}{ll}A & B \\ C & D\end{array}\right)$ in $S L(2, \mathbf{Z})$, symplectic and normalizing $\langle[\sigma],[\tau]\rangle$. The fact that a matrix is symplectic and normalizes $D_{4}$ gives a set of quadratic and linear equations from which we have found some particular integer solutions. The following symplectic matrix $f$, given by

$$
f=\left(\begin{array}{llll}
1 & 0 & 1 & 0 \\
0 & 1 & 0 & 1 \\
0 & 0 & 1 & 0 \\
0 & 0 & 0 & 1
\end{array}\right),
$$

satisfies $f[\sigma] f^{-1}=[\sigma], f[\tau] f^{-1}=[\tau]$, and the symplectic matrix $g$ given by

$$
g=\left(\begin{array}{cccc}
0 & 1 & -1 & 0 \\
0 & 1 & 0 & 0 \\
1 & 1 & 0 & 0 \\
-1 & 1 & -1 & 1
\end{array}\right),
$$

satisfies $g[\sigma] g^{-1}=[\sigma], g[\tau] g^{-1}=[\sigma][\tau]$. Then $f$ and $g$ define transformations of the modular group for $\Sigma_{1}$.

The complex structure of the space $\Sigma_{1}$ is given by the upper half-plane $\{t: \operatorname{Im} t>0\}$ via the map $t \rightarrow \frac{1}{2}\left(\begin{array}{ll}t & 1 \\ 1 & t\end{array}\right)$. Then the matrix $f$ induces on $\Sigma_{1}$ the transformation $V(t)=t+2$ and the matrix $g$ induces $W(t)=\frac{t-1}{t+1}$.

Let $H=\langle V, W\rangle$. Then $H$ is a subgroup of the modular group for our family. A fundamental region for the action of $H$ on $\{z: \operatorname{Im} z>0\}$ is in Figure 5 .

Then the quotient $\{z: \operatorname{Im} z>0, t \neq R(i), R \in \Gamma(2)\} / H$ is an orbifold of genus 0 , with one singular point of isotropy of order 2 and two cusps corresponding to $\infty$ and $i$.

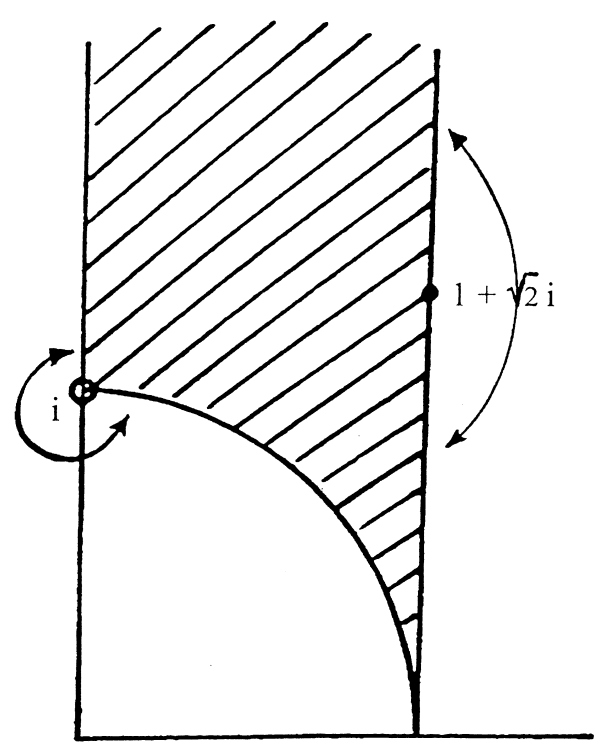

Figure 5 

result.

We shall show that in fact $H$ is the modular group and then prove the following

Proposition 4. The modular group for the family $F_{1}$ is generated by $V(t)=t+2$ and $W(t)=\frac{t-1}{t+1}$. The 1-complex orbifold structure for the moduli space is $O_{2, \infty, \infty}$ or, adding the elliptic curve $y^{2}=x(x+1)(x-1)^{3}$, the orbifold structure is $O_{2,4, \infty}$.

Proof. Assume that the modular group $M$ is different from $H$, so that $H<M$. Then we have an $n$-fold orbifold covering $p:\{t: \operatorname{Im} t>0, t \neq R(i), R \in \Gamma(2)\} / H \rightarrow$ $\{t: \operatorname{Im} t>0, t \neq R(i), R \in \Gamma(2)\} / M$.

At the beginning of the section we showed that $\{t: \operatorname{Im} t>0, t \neq R(i)$, $R \in \Gamma(2)\} / M$ is isomorphic to $O_{2, \infty, \infty}$ and so isomorphic as complex orbifold to $\{t: \operatorname{Im} t>0, t \neq R(i), R \in \Gamma(2)\} / H$. Thus $n=1$ and $H=M$.

We have remarked that the cone point of $O_{2, \infty, \infty}$ corresponds to the surface having automorphism group of order $48, X_{48}$. Hence $\frac{1}{2}\left(\begin{array}{cc}1+\sqrt{2} i & 1 \\ 1 & 1+\sqrt{2} i\end{array}\right)$ is the period matrix for $X_{48}$; (see [2]).

The orbifold structure of the moduli space of $F_{1}$ is given by the action on a connected equisymmetric stratus in the Teichmüller space by the action of the relative modular group. The stratus in the Teichmüller space is the fixed point set by the action of a finite subgroup of the modular group (given by the action of the automorphism group $D_{4}$ of the surfaces of the family). The relative modular group is the subgroup of the modular group preserving the stratus. Let $G_{24}$ be the automorphism group of the surface of the family with 24 automorphisms. If $f$ is an automorphism in $G_{24}-D_{4}$, then $f$ defines an element of the modular group but not in the relative modular group. The action of $f$ fixes the point corresponding to the Riemann surface with automorphism group $G_{24}$ but permutes three different strata in the Teichmüller space corresponding to surfaces with group of automorphisms conjugate to $D_{4}$.

The family of fixed matrices that we have is

$$
A=\left\{Z=\frac{1}{2}\left(\begin{array}{cc}
t & 1 \\
1 & t
\end{array}\right), \operatorname{Im} t>0\right\} .
$$

Let us act now via conjugation by an element of order 6 in the group of 24 automorphisms. The matrix of such an element in terms of the homology basis is

$$
[\rho]=\left(\begin{array}{cccc}
0 & 1 & -1 & 0 \\
1 & 1 & -1 & -1 \\
2 & 0 & -1 & -1 \\
0 & 2 & -1 & 0
\end{array}\right),[\rho]^{3}=-I .
$$

Now, the group $D_{4}$ generated by $[\sigma]$ and $[\tau]$ is not normal in the full group of automorphisms. We obtain

$$
[\rho]^{-1}[\sigma][\rho]=\left(\begin{array}{cccc}
0 & 1 & -1 & 0 \\
-1 & 0 & 0 & 1 \\
0 & 0 & 0 & 1 \\
0 & 0 & -1 & 1
\end{array}\right) .
$$


The matrices in Siegel's space fixed by this matrix are

$$
B=\left\{Z=\frac{1}{2}\left(\begin{array}{cc}
t & 1 \\
1 & \frac{t-1}{t}
\end{array}\right), \operatorname{Im} t>0\right\} .
$$

Furthermore, via conjugation by $\rho^{2}$ we obtain

$$
C=\left\{Z=\frac{1}{2}\left(\begin{array}{cc}
t & 1 \\
1 & \frac{1}{1-t}
\end{array}\right), \operatorname{Im} t>0\right\} .
$$

Thus $\frac{24}{\left|D_{4}\right|}=3$ corresponds to the three families $A, B, C$ being permuted by $\rho$. When we restrict ourselves to the family $A$ this action of order 3 disappears. The fixed point for the action of $\rho$ is the matrix $Z$, where $t=-\frac{1}{2}+\frac{\sqrt{3}}{2} i$, corresponding to the surface with automorphism group $G_{24}$.

4. Curves admitting real forms in the family $F_{1}$. The conjugation defines an anticonformal involution $\sigma^{*}$ on the moduli space of genus 2 surfaces; (see [15] and [17]). If $X$ is a surface of the family $F_{1}$ admitting an anticonformal involution $\sigma: X \rightarrow X$, then $\sigma$ defines an anticonformal involution $\tilde{\sigma}$ on the Teichmüller space $T_{2}$ (see [12] and [15]) and on the space of period matrices of the family $\Sigma_{1}$ (see [13]). The surface $X$ and the surfaces admitting an anticonformal involution with the topological type of $\sigma$ are in the fixed point set of $\widetilde{\sigma}$. The automorphism $\tilde{\sigma}$ is a lifting of $\sigma^{*}$. In the fixed point set of $\sigma^{*}$ there are the surfaces with anticonformal involutions.

There is a group $\widetilde{M}$ acting dianalytically (conformally-anticonformally) on $\Sigma_{1}$, such that the involutions $\widetilde{\sigma}$, for all anticonformal admissible involutions $\sigma$, are contained in $\widetilde{M}$ and the group $M$ is a subgroup of $\widetilde{M}$ of index 2. If $\theta$ is the map from $\mathbf{C}-\{-1,0,1\}$ to the family $F_{1}$ defined in Section 3 , then $\theta(\mathbf{R}-\{-1,0,1\})$ (that is an arc joining the two cusps) is contained in $\operatorname{Fix}\left(\sigma^{*}\right)$. The orbifold $O_{2, \infty, \infty}$ admits two anticonformal involutions but there is only one that preserves the cusps. (Note that the two cusps are different; one represents a stable curve with two nodes $(\mu=0)$ and the other is an elliptic curve $(\mu= \pm 1))$, the involution having as fixed point set two arcs joining the two cusps and one of the arcs passing through the cone point. Then $\widetilde{M}$ is the group generated by the reflections on the sides of the hyperbolic triangle with vertices $i, 1+\sqrt{2} i, \infty$. Thus the points in $O_{2, \infty, \infty}$, corresponding to surfaces having an anticonformal involution, are contained in the projection of the sides of such a triangle by $\pi: \Sigma_{1} \rightarrow O_{2, \infty, \infty}$. The projection of each side gives the points corresponding to surfaces with an anticonformal involution of a given topological type. We shall call:

Arc 1: The projection of the circular segment from $i$ to $1+\sqrt{2} i$; i.e. an arc in $\mathrm{O}_{2, \infty, \infty}$ joining the cone point with a cusp.

Arc 2: The projection of the imaginary axis from $i$ to infinity, i.e. an arc from one cusp to the other one.

$\operatorname{Arc} 3$ : The projection of the right line from $1+\sqrt{2} i$ to $\infty$, i.e. an arc in $O_{2, \infty, \infty}$ from a cone point to one cusp.

Before a case by case discussion we shall introduce some notation. If $X$ is a surface of the family we shall call $\operatorname{Aut}^{ \pm}(X)$ the group of conformal and anticonformal automorphisms of $X$. Let $X$ be a Riemann surface and $\tau$ be an anticonformal involution of $X$. We suppose that the fixed point set of $\tau$ consists of $k$ 
disjoint Jordan curves. Then the species of $\tau$ is defined to be $+k$ if $X /\langle\tau\rangle$ is orientable and $-k$ if $X /\langle\tau\rangle$ is nonorientable. The symmetry type of $X$ is defined to be the unordered list of the species of the conjugacy classes of anticonformal involutions of $X$.

Arc 1. By [3], the symmetry type $S_{1}$ of all the surfaces in Arc 1 that are not Platonic surfaces, must be one of the following ones: $\{-1,0,+1,+3\}$, $\{-2,-2,-1,-1\}$ or $\{-2\}$. Since the cone point is in Arc 1, the symmetry type $S_{1}$ must be contained as a subset in the symmetry type of $X_{48},\{-2,-1\}$. Then $S_{1}=\{-2,-2,-1,-1\}$ or $\{-2\}$. The point $i$ corresponds to the period matrix $\frac{1}{2}\left(\begin{array}{ll}i & 1 \\ 1 & i\end{array}\right)$ that is symplectically equivalent to a diagonal matrix [14]. Hence the point $i$ corresponds to an elliptic curve with period $i$. In terms of equations this corresponds to the case $\mu=\mu^{-1}=1$ or $\mu=\mu^{-1}=-1$. Then the surfaces corresponding to points in Arc 1 are given by $y^{2}=x\left(x^{2}-1\right)(x-\mu)\left(x-\mu^{-1}\right)$ with $\mu \bar{\mu}=1$. The anticonformal involutions in the surfaces $X$ in this arc are obtained by lifting the inversion on the unit circle and the reflection on the real axis. Then the orbifold $X / \operatorname{Aut}^{ \pm}(X)$ has a cone point in the interior, given by the projection of the fixed points of $x \rightarrow \frac{\mu x-1}{x-\mu}$. Thus the signature of an NEC group uniformizing $X / \operatorname{Aut}^{ \pm}(X)$ is $(0 ;+;[2] ;\{(2,4)\})$. Hence, by [3], the symmetry type $S_{1}$ is $\{-2\}$. In Figure 6 , it is shown how the group with signature $(0 ;+;\{(2,3,8)\})$ contains a subgroup of signature $(0 ;+;[2] ;\{(2,4)\})$, explaining how the surface $X_{48}$ appears at the end of this arc.

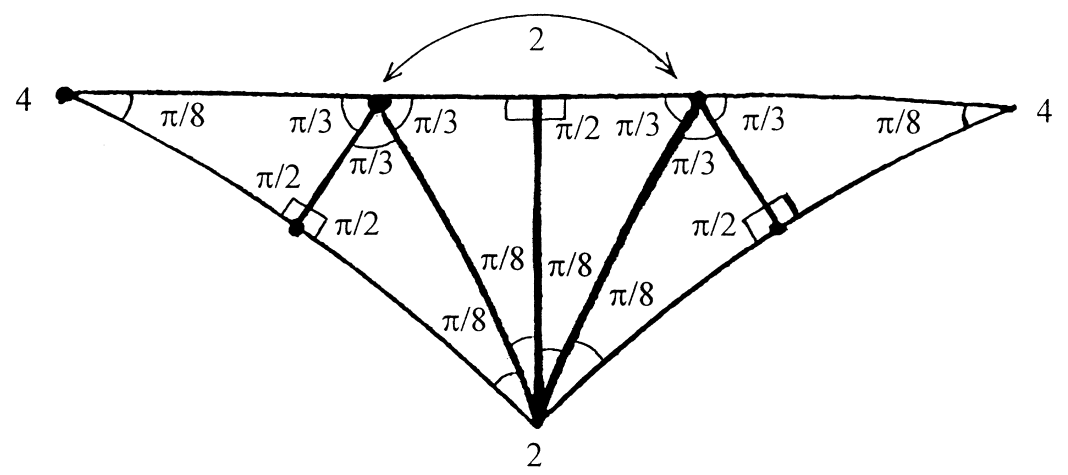

Figure 6

Arc 2. The Arc 2 joins the two cusps. Then the surfaces are the real algebraic curves $y^{2}=x\left(x^{2}-1\right)(x-\mu)\left(x-\mu^{-1}\right)$ with $\mu$ real. The symmetry type must be $\{-1,0,+1,+3\}$, (the unique symmetry type containing +1 ), because the symmetry type of $X_{24}$ is $\{-1,0,+1,+3\}$ and $X_{24}$ corresponds to $\mu=2$ or $\frac{1}{2}$. Figure 7 shows the way of inclusion of a group with signature $(0 ;+;[] ;\{(2,2,2,4)\})$ in one with signature $(0 ;+;[] ;\{(2,4,6)\})$, giving a geometrical way of detection of $X_{24}$ between the surfaces of the arc by the size of the Lambert quadrilateral $X / \operatorname{Aut}^{ \pm}(X)$.

Arc 3. The Arc 3 joins the surface with 48 automorphisms, $t=1+\sqrt{2} i$ or $\mu=i$ with the cusp represented by $t=\infty$ or $\mu=0$. Then the surfaces in Arc 3 are given by $y^{2}=x\left(x^{2}-1\right)(x-\mu)\left(x-\mu^{-1}\right)$ with $\operatorname{Re}(\mu)=0$. If $X$ is a surface in Arc 3 , the orbifold $X / D_{4}$ has an anticonformal involution with fixed point set a curve passing through the four cone points, and $X / \operatorname{Aut}^{ \pm}(X)$ can be uniformised by a group generated by the reflections on the sides of a Lambert quadrilateral with angles $\frac{\pi}{2}, \frac{\pi}{2}, \frac{\pi}{2}, \frac{\pi}{4}$. Then the signature of a group uniformizing $X / \operatorname{Aut}^{ \pm}(X)$ is $(0 ;+;[]$ 


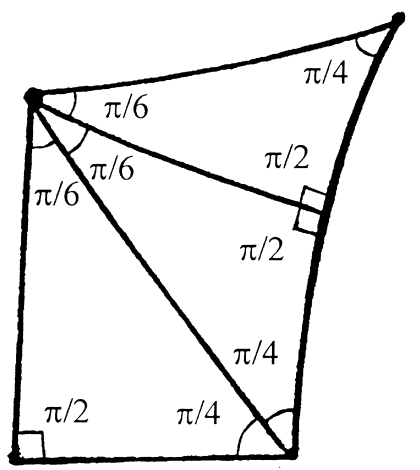

Figure 7

$\{(2,2,2,4)\})$ and then the symmetry type is $\{-2,-1\}$ (cf. [14]). In Figure 8 it is shown that the group of signature $(0 ;+;\{(2,3,8)\})$ contains an NEC group with signature $(0 ;+;[] ;\{(2,2,2,4)\})$. The surfaces in this arc can be parametrized by the hyperbolic cosine of the length $l$ of the bigger of the opposite sides to the vertex of the angle $\frac{\pi}{4}$ in the Lambert quadrilateral. For $\cosh l=\sqrt{2}$ (then $\cosh s=\frac{\sqrt{3}}{\sqrt{2}}$ ) the quadrilateral admits the decomposition of Figure 8 and then the surface corresponds to the Platonic surface of 48 automorphisms.

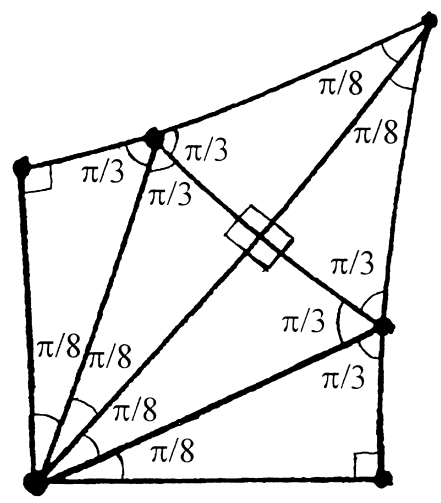

Figure 8

The real algebraic curves of the family $F_{1}$ has been also described by different methods in [4].

5. The family $y^{2}=x(x-1)(x-\lambda)\left(x-\frac{\lambda-1}{\lambda}\right)\left(x-\frac{1}{1-\lambda}\right), \lambda \neq \frac{1 \pm \sqrt{3} i}{2}, 0,1$. We shall call this family $F_{2}$. The Möbius transformation $x \rightarrow(x-1) x^{-1}$ lifts to an automorphism $\widehat{\sigma}$ of order 3 on each surface of the family $F_{2}$ and $x \mapsto(x-\lambda)((1-\lambda) x-1)^{-1}$ lifts similarly to an automorphism $\widehat{\tau}$ of order 2 . $\widehat{\sigma}, \widehat{\tau}$ and the hyperelliptic involution generate a group of automorphisms of order 12 isomorphic to $D_{3} \times C_{2}$.

This family also passes through the exceptional curves $\lambda=2$, the curve with 24 automorphisms and $\lambda=1-i$, the curve with 48 automorphisms.

The quotient of a surface of the family by $D_{3} \times C_{2}$ is an orbifold of genus 0 with four cone points with isotropy groups of orders $2,2,2$ and 3 . 
In a similar way to that for the family $F_{1}$ we obtain the following result.

Proposition 5. The Jacobian for $F_{2}$ is $\left\{Z=\left(\begin{array}{cc}s & -s / 2 \\ -s / 2 & s\end{array}\right) ; \quad \operatorname{Im} s>0\right.$, $\left.s \neq T\left(\frac{1}{2}+\frac{\sqrt{3}}{6} i\right), T \in M\left(F_{2}\right)\right\}$, where $M\left(F_{2}\right)$ is the modular group for $F_{2}$. Also $M\left(F_{2}\right)$ is a group generated by $s \rightarrow s+1, s \rightarrow \frac{-3 s-4}{3 s+3}$ and $s \rightarrow \frac{1}{-3 s+3}$.

A fundamental region for the modular group is in Figure 9.

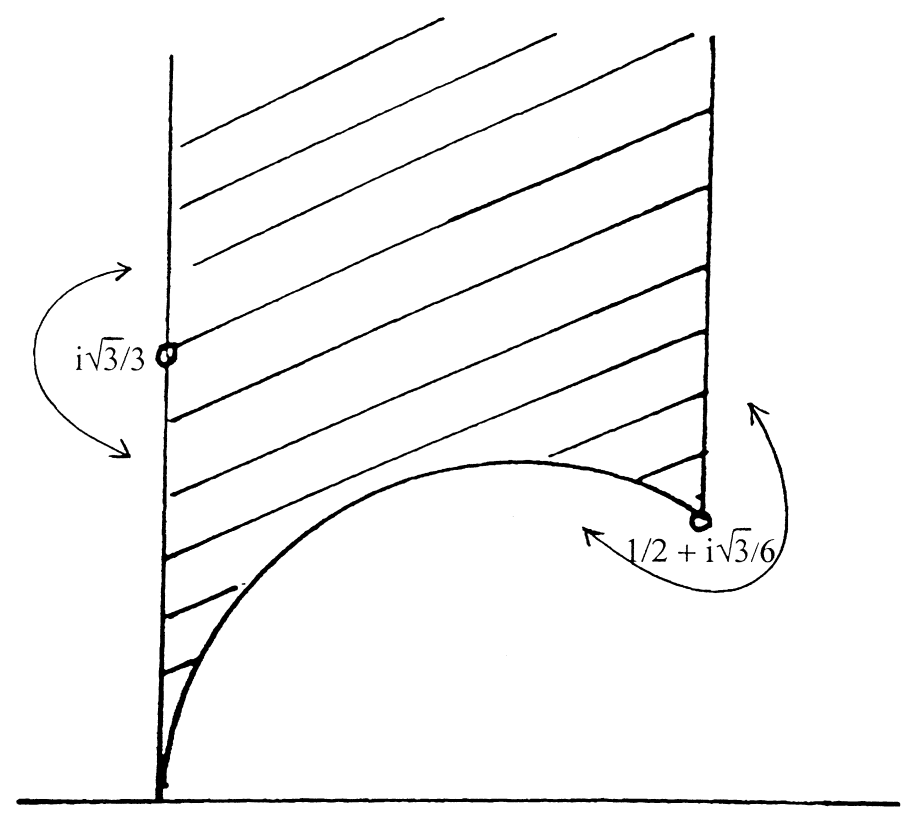

Figure 9

Proposition 6. The 1-complex orbifold structure of the moduli space $\Sigma_{2}$ for $F_{2}$ is $\mathrm{O}_{2, \infty, \infty}$ or, adding the elliptic curve corresponding to $s=\frac{1}{2}+\frac{\sqrt{3}}{6} i$, the orbifold structure is $\mathrm{O}_{2,6, \infty}$.

The point $s=\frac{\sqrt{3}}{3} i$ corresponds to the surface $X_{24}$ and the point $s=\frac{1}{2}+\frac{\sqrt{3}}{6} i$ corresponds to the elliptic Riemann surface with automorphisms group $D_{6}$.

The points in $O_{2, \infty, \infty}$ corresponding to surfaces with anticonformal involutions are in three arcs that are the projections by $\Sigma_{2} \rightarrow O_{2, \infty, \infty}$ of the sides of the hyperbolic triangle with vertices in $\frac{\sqrt{3}}{3} i, \frac{1}{2}+\frac{\sqrt{3}}{6} i$ and infinity.

The surfaces $X$, corresponding to the projection of the arc from $\frac{1}{2}+\frac{\sqrt{3}}{6} i$ to $\infty$, are such that $X / \operatorname{Aut}^{ \pm}(X)$ is a Lambert quadrilateral with angles $\frac{\pi}{2}, \frac{\pi}{2}, \frac{\pi}{2}, \frac{\pi}{3}$, i.e. the orbifold $X /\langle\widehat{\sigma}, \widehat{\tau}\rangle$ has an anticonformal involution passing through all cone points. The symmetry type for these surfaces is $\{-1,-1,0,+1\}$.

In the projection of the arc from $\frac{\sqrt{3}}{3} i$ to $\frac{1}{2}+\frac{\sqrt{3}}{6} i$ the orbifold $X /\langle\widehat{\sigma}, \widehat{\tau}\rangle$ has an anticonformal involution fixing the cone point of the isotropy group of order 3 and only one of the singular points with isotropy $C_{2}$. The corresponding surfaces have symmetry type $\{-1\}$. In this arc appears the Platonic surface $X_{48}$. 

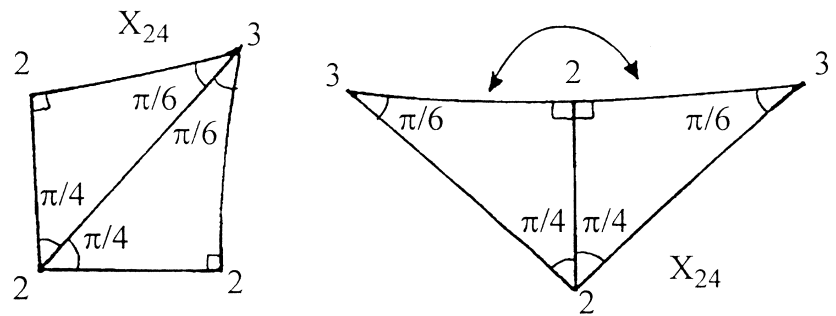

Figure 10

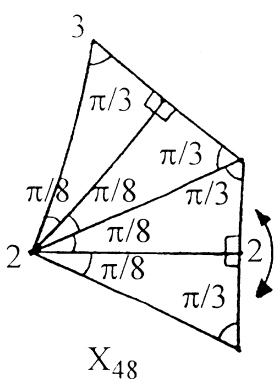

Also, in the projection of the arc from $\frac{1}{2}+\frac{\sqrt{3}}{6} i$ to $\infty$, the orbifold $X /\langle\widehat{\sigma}, \widehat{\tau}\rangle$ has an anticonformal involution with fixed point set a closed curve passing through all cone points. The corresponding surfaces have symmetry type $\{0,+1,+3,+3\}$.

If we call $c$ the anticonformal involution of $X /\langle\widehat{\sigma}, \widehat{\tau}\rangle$ described above for $X$ a surface in the arcs, the decomposition of $X /\langle\widehat{\sigma}, \widehat{\tau}\rangle /\langle c\rangle$ in the points corresponding to Platonic surfaces is shown in Figure 10.

REMARK 7. If we add to $F_{1}$ and $F_{2}$ the two elliptic curves in the boundary of the moduli space of such families, the 1-complex orbifold structure of the moduli space can be uniformized in the first case by the Hecke group $(2,4, \infty)$ and in the second case by $(2,6, \infty)$ and for the moduli space of elliptic curves by $(2,3, \infty)$. D. Singerman pointed out that $(2,4, \infty),(2,6, \infty)$ and $(2,3, \infty)$ are exactly the maximal arithmetic groups between the Hecke groups.

Acknowledgements. The authors wish to thank the referee for pointing out the fact that period matrices of curves can be computed numerically using algorithms included in the new package algcurves in Maple V.6 [6]. These algorithms apply particularly well to examples in the families studied in this work.

The authors are grateful to Emilio Bujalance, Javier Cirre, José M. Montesinos, S.M. Natanzon, Robert Silhol and Peter Turbek for helpful discussions during the preparation of this paper.

\section{REFERENCES}

1. N. Alling, Real elliptic curves (North-Holland Publishing Company, 1981).

2. O. Bolza, On binary sextics with linear transformations into themselves, Amer. J. Math. 10 (1888), 47-60.

3. E. Bujalance and D. Singerman, The symmetry type of a Riemann surface, Proc. London Math. Soc. (3) 51 (1985), 501-519.

4. P. Buser and R. Silhol, Geodesics, periods and equations of real hyperelliptic curves, Preprint 1998.

5. P. du Val, Elliptic functions and elliptic curves, London Mathematical Society, Lecture Note Series 9 (Cambridge University Press, 1973).

6. B. Deconinck and M. van Hoeij, Computing Riemann matrices of algebraic curves, Preprint 1999, available at www.math.fsu.edu/ hoeij.

7. C. J. Earle, H. E. Rauch, function theorist, in Differential geometry and complex analysis, H. E. Rauch Memorial Volume (Springer-Verlag, 1985), 15-31.

8. G. A. Jones and D. Singerman, Theory of maps on orientable surfaces, Proc. London Math. Soc. (3) 37 (1978), 273-307. 

1987).

9. G. A. Jones and D. Singerman, Complex functions (Cambridge University Press,

10. R. Kulkarni, Isolated points in the branch locus of the moduli space of compact Riemann surfaces, Ann. Acad. Sci. Fenn. Ser. A. I. Math. 16 (1991), 71-81.

11. T. Kuusalo and M. Näätänen, Geometric uniformization in genus 2, Ann. Acad. Sci. Fenn. Ser. A. I. Math. 20 (1995), 401-418.

12. A. M. Macbeath and D. Singerman, Spaces of subgroups and Teichmüller spaces, Proc. London Math. Soc. (3) 31 (1975), 211-256.

13. G. Riera, Automorphisms of abelian varieties associated with Klein surfaces, $J$. London Math. Soc. (2) 51 (1995), 442-452.

14. J. Schiller, Moduli for special Riemann surfaces of genus 2, Trans Amer. Math. Soc. 144 (1969), 95-113.

15. M. Seppälä, Complex algebraic curves with real moduli, J. Reine Angew. Math. 387 (1988), 209-220.

16. R. Silhol, Normal forms for period matrices of real curves of genus 2 and 3, J. Pure Appl. Algebra 87 (1993), 79-92.

17. M. Seppälä and R. Silhol, Moduli spaces for real algebraic curves and real abelian varieties, Math. Z. 201 (1989), 151-165. 